\title{
CoviD-19 The value of healthcare worker support strategies to enhance wellbeing and optimise patient care
}

\author{
Authors: Sarah Diver, ${ }^{\mathrm{A}}$ Nicola Buccheri ${ }^{\mathrm{B}}$ and Chandra Ohri ${ }^{\mathrm{C}}$
}

\section{Introduction and aim}

The World Health Organization has recognised the impact of the SARS-CoV-2 pandemic on healthcare worker (HCW) mental health and wellbeing. Anticipating these effects locally, we developed strategies to support our team, to equip them to care for themselves as well as our patients.

\section{Methods}

We implemented a series of interventions to increase staff support, highlighting the importance of team and individual morale. We developed a team of peer supporters, encouraged sub-teams to debrief and disseminated general wellbeing advice.

Results

Feedback demonstrates that our interventions had a positive impact. Greater benefits were recognised by empowering sub-teams to develop their own wellbeing and support mechanisms.

\section{Conclusion}

A strategy to support HCW teams during a crisis is vital to enhance wellbeing. Interventions implemented within our team have supported the provision of high-quality patient care, innovation and research throughout the pandemic.

KEYWORDS: COVID-19, wellbeing, support, healthcare worker

DOI: $10.7861 /$ fhj.2020-0176

\section{Introduction}

Severe Acute Respiratory Syndrome Coronavirus 2 (SARS-CoV-2), also known as COVID-19, emerged from Wuhan, China, in late 2019, rapidly leading to an international pandemic. As of January 2021, there have been over 86 million cases globally, and over 1.8 million deaths. ${ }^{1}$ One article from Singapore demonstrates the initial psychological impact of the pandemic,

Authors: ${ }^{A}$ clinical research fellow and respiratory ST6 doctor, Glenfield Hospital, Leicester, UK and University of Leicester, Leicester, UK; ${ }^{B}$ Clinical psychologist, Leicestershire Partnership NHS Trust, Leicester, UK; 'consultant respiratory physician and honorary associate professor of medical education, Glenfield Hospital, Leicester, UK and University of Leicester, Leicester, UK reporting that $25 \%$ of people have experienced at least moderate anxiety symptoms. ${ }^{2}$ Increased global connectedness and media coverage may contribute to the increased mental health burden of SARS-CoV-2 on individuals compared to previous pandemics. This burden is further increased in healthcare workers (HCWs), and with successful delivery of healthcare in part dependent on their morale and wellbeing, ${ }^{3}$ specific safeguarding of mental health in this group has been recommended internationally. ${ }^{4}$

In the UK, as of 7 January 2020, there have been 2,889,419 confirmed cases and 78,508 deaths, ${ }^{5}$ including a reported 272 deaths in HCW aged $20-64^{6}$ during the first wave, with significantly increased risk in males, and across gender in specific occupations including nursing. It is well recognised that poor staff health and wellbeing has a negative impact on the performance of NHS organisations: ${ }^{7}$ however, the immensely pressured working environment faced by staff on the frontline throughout the coronavirus pandemic has led employees at NHS trusts across the country to acknowledge their wellbeing as a critical priority. There are many risks to HCW wellbeing, including allocation of finite resources and the difficulty of balancing the desire to care for patients against responsibilities to protect their physical health and that of their family and friends. ${ }^{8}$ Many trusts have redistributed staff to support the frontline, and redeployment away from the usual work environment or parent specialty can potentially lead to feelings of uncertainty and loss of confidence.

Glenfield Hospital in Leicester is one of three sites making up the University Hospitals of Leicester NHS Trust (UHL), one of the biggest and busiest trusts in the country, serving a highly diverse population of 1 million residents across Leicester, Leicestershire and Rutland, extending further to 2,785,083 million that access specialist services, including cardiorespiratory disease services and extracorporeal membrane oxygenation. UHL employs over 16,000 staff in total, with over a third from a Black, Asian and minority ethnic (BAME) background. Glenfield Hospital hosts tertiary services for respiratory medicine, including 129 inpatient beds and a large respiratory multidisciplinary team (MDT). Specific strategies to combat the SARS-CoV-2 pandemic were implemented at Glenfield Hospital site in March 2020. Many HCWs in our Trust closely followed the development of the pandemic in other nations, and there was significant anxiety that the NHS could be overwhelmed.

Staff wellbeing is a key priority for UHL, with a dedicated Trust wellbeing team involved in a range of interventions, including 
providing regular email support to staff across the Trust during this crisis. Given the size of the MDT and the anticipated level of SARS-CoV-2-related activity within our department, it was felt important to develop a more personal strategy. The World Health Organization (WHO) issued guidance for those managing HCW on protecting from the effects of poor mental health and chronic stress, including delivery of high-quality accurate communication, promoting social support and facilitating access to mental health services. ${ }^{4}$

In this report, we share our experience of additional local wellbeing support at the Glenfield Hospital site, and suggest good practice points that colleagues in other centres may find useful. Further practical advice on implementing these interventions is available in the supplementary material (S1).

\section{Implementation of our strategy}

\section{Strategy overview}

It was recognised that the needs of individual staff members varied, and would change over time; therefore interventions were introduced in parallel to support staff with lesser to greater psychological needs, following the stepwise response outlined by the British Psychological Society ${ }^{9}$ and Inter-Agency Standing Committee (IASC). ${ }^{10}$ Key components of our strategy were:

$>$ to provide ways to support colleagues with varying needs

$>$ to offer advice on reducing stress and anxiety both for individuals and teams

$>$ to develop resources to help support colleagues and peer supporters

$>$ to improve morale and recognise the significant contributions of individuals and teams

$>$ to escalate individual cases to appropriate healthcare professionals as required.

\section{Developing a team}

A senior respiratory clinician with experience in clinical education and mentoring roles coordinated staff wellbeing. A core wellbeing group developed, engaging specific members of the clinical and academic MDT with relevant skills, including clinical psychology, supported by input from other staff with key perspectives.

\section{Stage 1: Departmental briefing - 'Supporting each other'}

Pandemic response strategies were communicated to the workforce at an initial briefing. Parallels had been highlighted between the potential moral injuries that could be experienced by clinicians managing the pandemic and those experienced by military personnel participating in campaigns, ${ }^{8}$ and, innovatively, an exmilitary respiratory consultant within the department was invited to provide advice on maintaining resilience. Protecting staff wellbeing was identified as a key component of the overall response. The wellbeing lead clearly identified himself and outlined the approach to staff support, included the provision of basic resources drawing on the principles of psychological first aid, ${ }^{11}$ recommending all staff to ask each other two daily questions ('Am I OK?' and 'Are you OK?') and introducing morale boosting and peer support strategies. In addition to the military comparisons drawn above, the principles of crisis resource management, evolved from the aviation industry, were felt applicable and were highlighted to staff (Fig 1). 12,13 As some were not able to attend the briefing, the wellbeing messages were recorded into a video entitled 'Health and wellbeing' and made accessible online.

\section{Stage 2: Development of our peer supporter team}

Peer support is a helpful tool for clinicians to improve staff wellbeing through the sharing of experiences and strategies with colleagues, either in the form of team debriefs or one-on-one

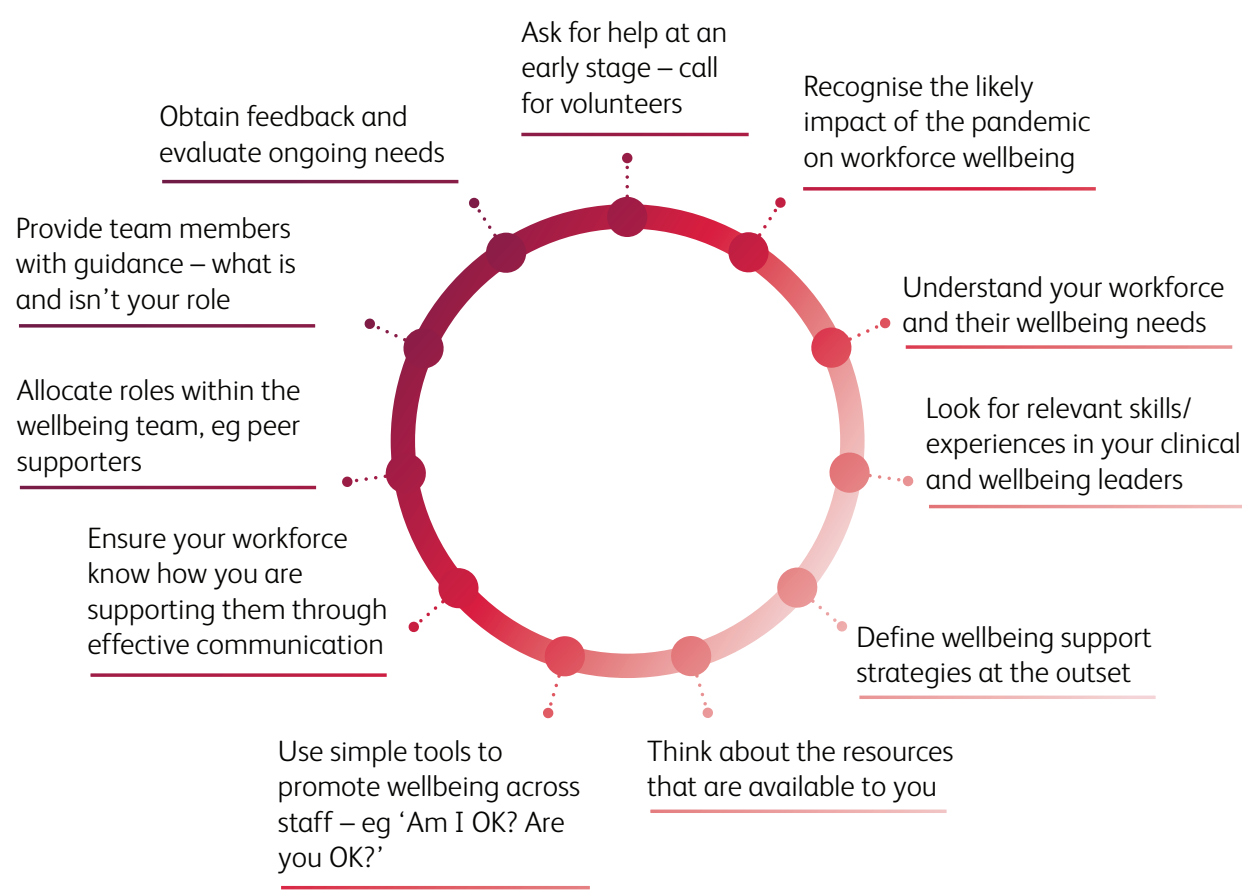

Fig 1. Key point of crisis resource management $(\mathrm{CRM})^{13}$ as applied at our site. 


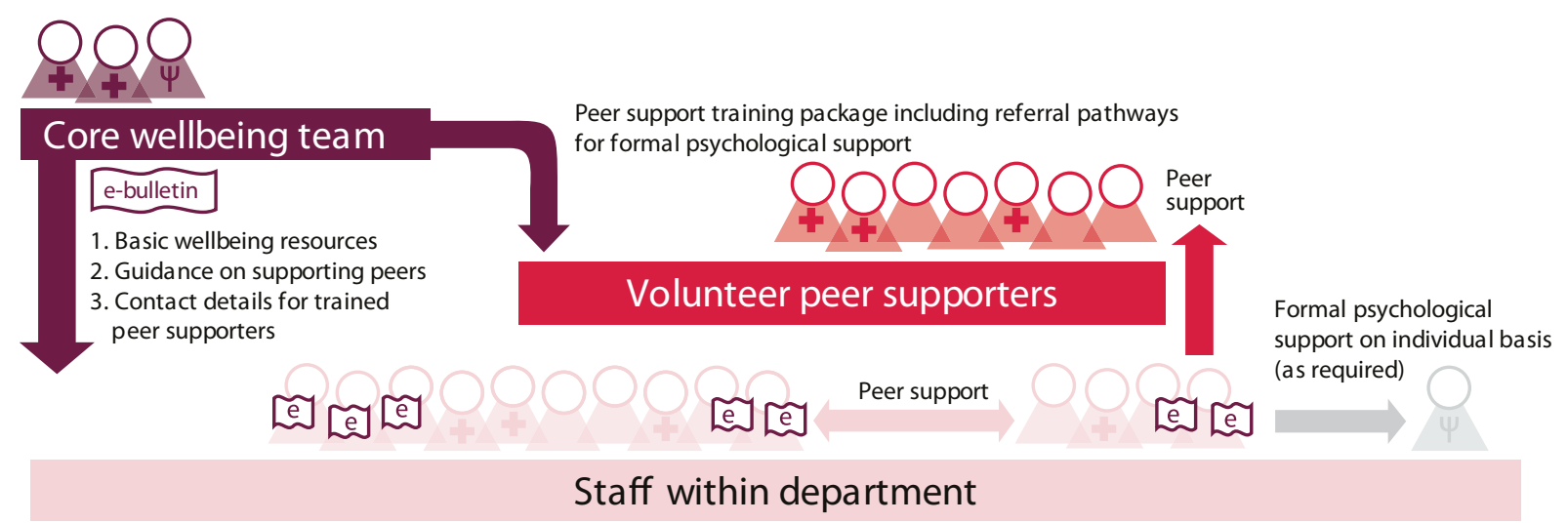

Fig 2. Healthcare professionals involved in wellbeing and peer support.

interactions. ${ }^{14}$ We formed a team of volunteer peer supporters with an interest in wellbeing at the outset of the pandemic. The core wellbeing team promoted the role and offered guidance at the initial briefing. Following calls for volunteers over several weeks, membership increased to 22. Members are from different specialities and disciplines, including individuals in non-clinical roles, with strong allied healthcare professional and academic representation, and representation across levels of seniority from foundation grade to directorate clinical director.

Team members shared the key objective of breaking down traditional hierarchies of seniority and encouraging staff members at all levels to consider accessing peer support to maintain wellbeing (Fig 2). Online training was provided to peer supporters outlining the aims of the role, with a focus on listening without judgement, providing objective and rational advice where appropriate and encouraging staff to take responsibility for their own wellbeing. It was highlighted that peer support was in no way a replacement for normal mechanisms of healthcare such as those provided by GPs, occupational health, formal counselling services or mental healthcare professionals, and formal referral pathways were clearly defined. An e-resource pack was provided to peer supporters, which included guidance on peer support techniques, along with resources to share with staff relating to the specific domains of worry and anxiety, sleep, physical activity and diet.

Stage 3: Morale boosting and wellbeing advice: weekly e-bulletin

To boost morale, an 'Inspiration of the week' scheme was commenced, welcoming staff to highlight the significant contributions made by their colleagues throughout the pandemic, recognising that there would be positive outcomes despite the challenges. This has proved so popular that there are several nominations for individuals and teams on a weekly basis.

With high volume email communications initiated during the pandemic, we felt that further daily email communication would have been excessive. As a result, we implemented a weekly e-bulletin. This allowed provision of support across psychological needs in one A4-size publication, containing contact details for peer supporters for those with greater or increasing needs, alongside the 'Inspirations of the week' and basic resources for maintaining physical and emotional wellbeing (Box 1).

\section{Box 1. Wellbeing information and resources promoted} by the weekly e-bulletin during the pandemic

Signposting to resources

Apps (supporting eg mental health, sleep, physical activity) Podcasts

Online videos

Rest spaces (eg on-site garden, ward 'Novid' spaces, site 'Novid' zones) Articles

Hashtags (eg \#StayInWorkOut from Sport England)

Counselling helplines, including those focusing on specific issues such as grief

Websites (eg Intensive Care Society wellbeing resource library, NHS staff benefits)

Details of local volunteer groups

'Recovery from illness' therapy leaflets

Results of research trials that the Trust had participated in

\section{Morale boosters}

'Inspiration of the week'

Patient feedback

Family messages

Positive reflections (peer supporters were asked to reflect on their positive outcomes from the pandemic)

'PositiviTrees' - walls of clinical areas decorated with positive messages

Inspirational quotes and pictures

Tips to improve aspects of wellbeing

Managing anxiety and worry (eg rescue breathing, keeping track of stressors)

Enhancing sleep

Maintaining physical activity

Making decisions

Working in masks

Staying connected with family and friends

Regulating exposure to social media

Supporting colleagues

Avoiding moral fatigue

Teambuilding and individual exercises

The COVID-19 Mixtape Exchange (team members sharing a 10-song playlist)

Team debriefs - virtual or social distanced, ground rules provided Gratitude diaries 
We felt it was important to recognise staff for a broad spectrum of achievements for 'Inspiration of the week', whether they had impact locally and nationally, or simply improved the quality of working life for staff within their team. Examples included the following:

> Exemplar leadership, particularly in busy clinical areas, eg staff involved in designing and implementing SARS-CoV-2 follow up services.

$>$ Communication, eg the intensive care support team contacting relatives every day with updates.

> Innovation, eg those implementing the SPACES ${ }^{15}$ approach, or the physiotherapists and specialist nurses involved in the formation of a new acute respiratory response team (ARRT) to CO-ordinate respiratory support in SARS-CoV-2 patients.

> Research leadership and delivery, eg recognising the achievements of staff and investigators working on SARS-CoV-2 clinical trials. ${ }^{16}$

> Improved ward environment, eg a medical trainee publishing and distributing a book of children's drawings to cheer up inpatients affected by restricted visiting.

> Light relief, eg a staff member taking on a 'quizmaster' role in his spare time, creating weekly quizzes for colleagues to enjoy.

\section{Stage 4: Empowering teams to support each other}

The e-bulletin was used to empower teams to support each other in different and novel ways (Box 1), with colleagues encouraged to strengthen relationships within their teams and ensure that connectedness was maintained despite physical distancing. We felt that it was important for individual teams (usually wardbased or sub-specialty MDTs) to take ownership of this concept, depending on their own feelings, needs and attitudes.

Debriefing is a technique intended to reduce psychological morbidity and the development of long-term complications following traumatic episodes. With military origins, debriefs aimed to maintain group morale and reduce distress among soldiers post-combat. ${ }^{17}$ While single-session, early individual debriefs are contraindicated due to the risk of increasing long-term morbidity, ${ }^{17}$ group debriefing has been shown to improve short-term overall quality of life and reduce alcohol abuse. ${ }^{18}$ Modified techniques focusing on positive changes relating to the event rather than recounting of the event have similarly led to improved outcomes in soldiers, ${ }^{19}$ although it is unclear whether the positive impact of group debriefing may simply reflect the overall benefits of social support. Additionally, team forums led by clinical leaders, in which staff are encouraged to make sense of morally challenging decisions, could help to reduce the risk of moral injury. ${ }^{8}$

'Shop floor' clinicians were encouraged to trial debriefing sessions within their teams, either in socially distanced spaces or online. Wellbeing team members role-modelled the technique to encourage participation and highlight common pitfalls. Keys to successful sessions included identification of a chair and using positive language to avoid causing anxiety. Once this was rolled out across clinical areas it was recognised that each ward team/MDT had their own preferences, such as varying degrees of formality and frequency.

Increased and diversified communication was encouraged, and some chose to use platforms such as WhatsApp groups during the pandemic. One example of this is a respiratory senior clinician group set up with three main aims:

> To request urgent support if overwhelmed by cases or due to staff sickness.
> To seek an urgent second opinion for challenging and ethical decisions.

> The rapid dissemination of breaking clinical updates, such as latest patient management guidelines relating to SARS-CoV-2, or strategy updates.

\section{Feedback}

Although Leicester's recovery from the pandemic had been complicated by a resurgence of cases after the initial peak, ${ }^{20}$ SARS-CoV-2-related workload at our site declined through August, allowing staff time to reflect on their experiences. To obtain feedback and to assess ongoing needs we circulated a survey to 392 staff receiving the e-bulletin. Interventions were rated on a Likert scale from 'extremely useful' to 'not at all useful', with free text for comments relating to specific interventions. 39 staff members completed the survey. Feedback is presented in Fig 3. Interestingly, although $86 \%$ of responders found it useful to know that the peer supporters were available, as was also reflected in narrative feedback, the group were rarely contacted. Two staff members highlighted that the process of seeking out help could deter some individuals. The peer supporters provided ad hoc support more frequently, and found that individuals felt more supported within teams where rapport and relationships were established before the pandemic.

While debriefing sessions met with positive informal feedback, uptake of these sessions was variable. This may reflect that individuals engaging with these interventions were more likely to provide feedback. Leaders of remote debriefing sessions reported finding these to be useful for communicating updates and checking in on team members.

Staff reported an appreciation for the brevity of our e-bulletin and the weekly distribution, with most responders suggesting that these should continue at a reduced frequency after the first wave. Participation in work-related group chat platforms received extremely mixed feedback (Fig 4).

\section{Discussion}

Overall, additional interventions to support staff during the coronavirus pandemic had a positive impact on wellbeing. Staff appreciated communication tools that were targeted and highly relevant, whilst informal peer support within teams appeared to be more useful than formal support sessions.

Analysis of recent coronavirus pandemics, including SARS-

CoV-2, highlights the risk of HCW developing post-traumatic stress disorder (PTSD) as a result of their occupation. ${ }^{21}$ Moral injury may also occur, which can contribute to the development of conditions such as PTSD, depression and anxiety. ${ }^{22}$ Accurately evaluating the impact of crisis episodes is critical as treatment approaches vary, and the development of mental health conditions in the workforce may affect the ability to provide optimal patient care post-crisis. The value of social media in 'educating at scale' is increasingly recognised ${ }^{23}$ and many of our workforce utilised these platforms during the pandemic to connect with and learn from colleagues locally and nationally. However, the WHO have described the media availability of COVID-19 related information an 'infodemic' with potentially negative consequences, ${ }^{24}$ reflected in other studies from Europe and China reporting positive correlation between media exposure and mental health symptoms. ${ }^{25,26}$ 
Was the initial briefing useful?

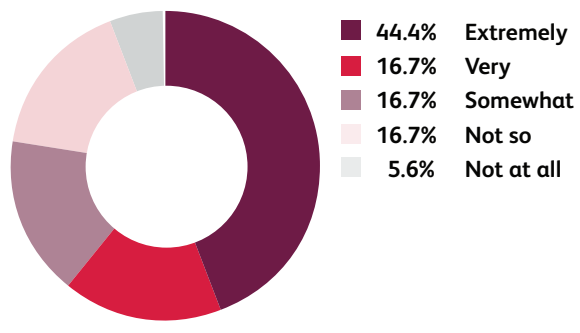

20 respondents did not attend the briefing

Were debriefing sessions useful?

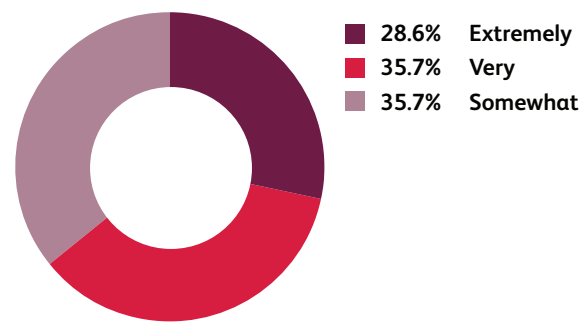

Eight respondents did not participate in debriefing sessions
Was it useful to know that peer supporters were available?

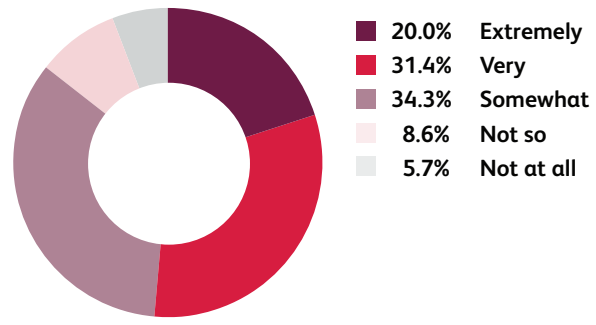

'Sometimes even when help is made available, it is still hard to ask for, especially when you know the people offering it are busy'

\section{Did you participate in a remote work-related group chat during the pandemic?}

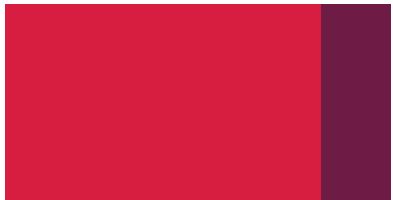

$\mathbf{8 1 . 1 \%}$ Yes

- $18.9 \%$ No

Was the e-bulletin useful?

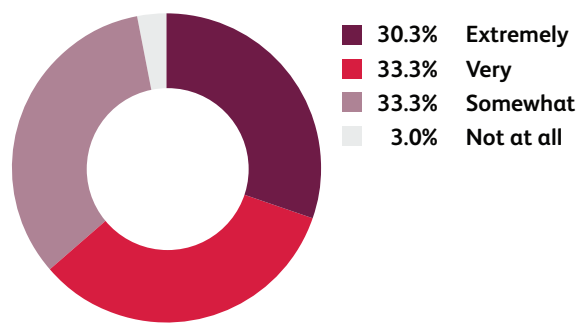

Four respondents had not looked at the e-bulletin

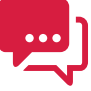

'The e-bulletin was useful for knowing that support was there'

'The positive praise of staff was the highlight'

'The resources that were shared were useful'
Would you like to see the e-bulletin continue?

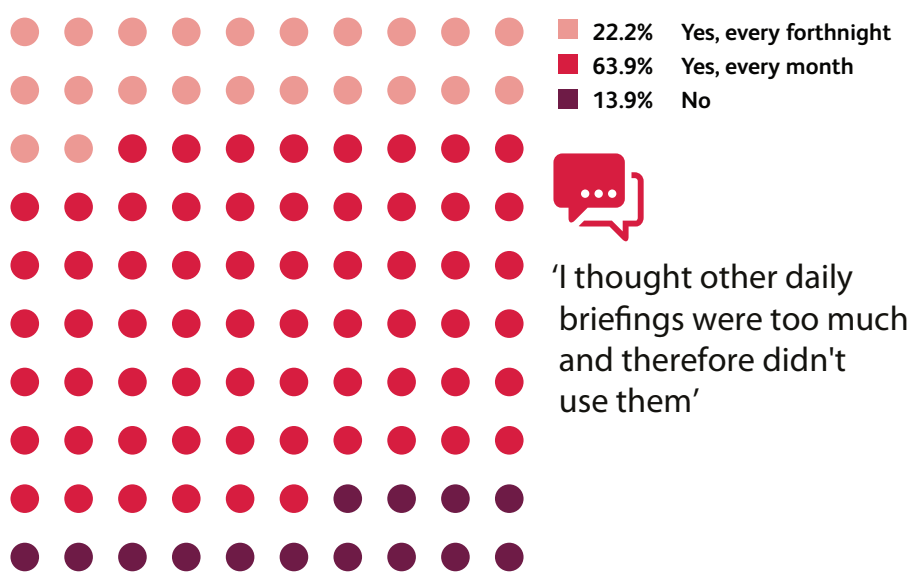

Fig 3. Results of feedback on local wellbeing support interventions.

While it is recognised that differences in the needs of individual staff will lead to varying responses to interventions, a 'one-sizefits-all' approach has been the most utilised to psychological support for HCW exposed to disasters, due to limited time to design tailored support programmes in advance. Our staff did not seek help from designated peer supporters, which could indicate that they were not useful; however, feedback suggests that HCW appreciated knowing that these systems were in place. Others suggested that our peer support intervention was not suitably proactive. The majority of staff are likely to feel adequately supported through non-targeted strategies but it is clearly important to identify those with greater needs. How best to approach this situation is unclear; some HCWs may feel stigmatised if concerns about them are highlighted, while taking a blanket approach of increased support may lead others to feel burdened by interventions they consider unnecessary. Our solution has been to cascade the peer support training across the workforce via the e-bulletin, to ensure that HCWs feel confident to offer support within their teams. Autonomy over the bulletin content has allowed us to respond to feedback in real time. 


\section{Work-related group chat platforms}

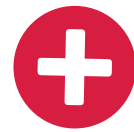

Advantages

$>$ Rapid dissemination of information

$>$ Improve team and personal morale

$>$ Share important resources

$>$ Communicate with colleagues off-site

$>$ Use to request additional support if needed due to eg sickness, high caseload

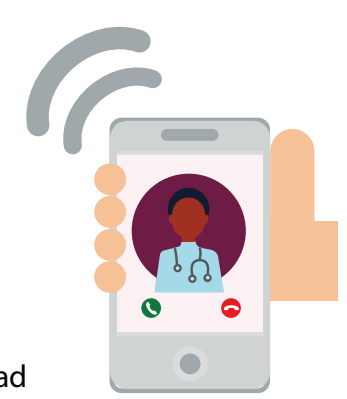

Do:

$>$ Respect the group purpose

$>$ Only use for information of relevance to

the majority - use direct messaging to

contact a specific person

$>$ Nominate a moderator to keep the group

'on purpose'

$>$ Use the 'reply' function to respond to

specific comments or requests

$>$ Treat all group members with respect

$>$ Minimise out-of-hours messaging

Fig 4. Narrative feedback relating to work-related group chat platforms.

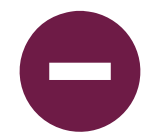

Disadvantages

> Out-of-hours time disrupted, preventing effective down-time and raising anxiety

$>$ Risk of negative or unsupportive comments

$>$ Team members may have different levels of need

$>$ Information may be irrelevant

$>$ Hard to follow different agendas in one group

Don't:

$>$ Use to discuss divisive issues - tone and meaning can be misconstrued

$>$ Share content without verifying the source - misinformation can be harmful to wellbeing

$>$ Judge those who leave or mute the group

$>$ Deviate from Trust policy on use of social media
Workplace group chat platforms were clearly useful for disseminating information in the setting of rapidly evolving clinical practice but met with significant negative feedback (Fig 4). We suggest implementing ground rules at the outset to allow staff access to the benefits while protecting from the negative effects.

Collecting representative feedback on our interventions has been challenging. We achieved a response rate of approximately $10 \%$ from our survey, supported by informal feedback from staff and peer supporters. Pre-pandemic, face-to-face meetings were often a useful opportunity to engage staff; however, departmental meetings are now conducted remotely for infection control. We felt that repeated email reminders to complete feedback may have placed additional burden on staff with ongoing stressors; however, we recognise that this may mean important perspectives have been overlooked. Although we did not collect information relating to other indicators of wellbeing, such as alcohol use or access to counselling, the increased anonymity of remote feedback may allow staff to be feel less vulnerable in disclosing this important information within their organisation.

So what are the future plans for the wellbeing interventions that were employed during the pandemic? Staff clearly indicated they would like some aspects of wellbeing support to continue, for example an e-bulletin at a reduced frequency. Wellbeing resources may become less relevant as caseload falls, but fostering relationships within teams remains critically important. It is well recognised that frequent rotation of staff in clinical placements can lead to superficial team relationships and increase stress ${ }^{27,28}$ and the way in which teams have considered their collective skills and worked together to protect each other (eg SPACES ${ }^{15}$ ) is a consistent positive reflection reported by staff during the pandemic. We found that empowering smaller teams to support individual members resulted in a greater sense of engagement and wellbeing compared to a corporate approach of 'one model for all'. We aim to continue to use the e-bulletin to highlight team achievements and encourage teambuilding exercises. There are also plans to distribute a similar bulletin to undergraduate students on clinical placement at the Trust.

\section{Conclusion}

We found that wellbeing interventions impacted positively on HCWs at our site. This may have augmented the delivery of highquality patient care, including the innovation and research which has been recognised internationally. Sharing and reflecting on our experiences may be helpful for colleagues in other healthcare settings who plan to implement wellbeing strategies in the context of the ongoing pandemic or in response to other crises in future.

\section{Supplementary material}

Additional supplementary material may be found in the online version of this article at www.rcpjournals.org/fhj: S1 - A blueprint for wellbeing support strategies for clinical teams during the SARS-CoV-2 pandemic and future crises.

\section{Acknowledgements}

We thank the wider team of volunteer Peer Supporters at Glenfield Hospital, and all staff members who engaged with the wellbeing support strategies and provided feedback. 


\section{References}

1 World Health Organization. WHO Coronavirus Disease (COVID-19) Dashboard. https://covid19.who.int/ [Accessed 8 January 2021].

2 Ho CS, Chee CY, Ho RC. Mental health strategies to combat the psychological impact of COVID-19 beyond paranoia and panic. Ann Acad Med Singapore 2020;49:155-60.

3 Blake H, Bermingham F. Psychological wellbeing for healthcare workers: mitigating the impact of Covid-19 (version 2.0). University of Nottingham, 2020. www.nottingham.ac.uk/toolkits/play_22794.

4 World Health Organization. Mental health and psychosocial considerations during the COVID-19 outbreak. WHO, 2020. www.who. int/publications/i/item/mental-health-and-psychosocial-considerations-during-the-covid-19-outbreak.

5 Public Health England. Coronavirus (COVID-19) in the UK. https:// coronavirus.data.gov.uk/ [Accessed 8 January 2021].

6 Office for National Statistics. Coronavirus (COVID-19) related deaths by occupation, England and Wales: deaths registered between 9 March and 25 May 2020. ONS, 2020. Available from www.ons. gov.uk/peoplepopulationandcommunity/healthandsocialcare/ causesofdeath/bulletins/coronaviruscovid19relateddeathsbyoc cupationenglandandwales/deathsregisteredbetween9marchand25may2020\#overview-of-coronavirus-related-deaths-by-occupation.

7 NHS Employers. Workforce health and wellbeing framework. NHSE, 2019. Available from www.nhsemployers.org/case-studies-andresources/2018/05/nhs-health-and-wellbeing-framework.

8 Greenberg N, Docherty M, Gnanapragasam S, Wessely S. Managing mental health challenges faced by healthcare workers during covid-19 pandemic. BMJ 2020;368:m1211.

9 British Psychological Society Covid-19 Staff Wellbeing Group. The psychological needs of healthcare staff as a result of the Coronavirus pandemic. British Psychological Society, 2020.

10 Inter-Agency Standing Committee Reference Group on Mental Health and Psychosocial Support in Emergency Settings. Interim briefing note: addressing mental health and psychosocial aspects of COVID-19 outbreak. IASC, 2020.

11 COVID Trauma Response Working Group. COVID Trauma Response Working Group clinical guidance: psychological first aid. CTRWG, 2020. Available from www.traumagroup.org.

12 Gaba DM. Crisis resource management and teamwork training in anaesthesia. Br J Anaesth 2010;105:3-6

13 Fanning RM, Goldhaber-Fiebert SN et al. Crisis resource management. In: Levine AI, DeMaria S, Schwartz AD, Sim AJ (eds), The Comprehensive Textbook of Healthcare Simulation. New York, NY: Springer, 2013:95-109.

14 Nadel AL, Barbach N, Fish JD. How I approach peer support in pediatric hematology/oncology. Pediatr Blood Cancer 2020;67:e28297.

15 British Thoracic Society. Add SPACES to your COVID ward care approach. BTS, 2020.

16 RECOVERY: Randomised Evaluation of COVID-19 Therapy. www. recoverytrial.net/ [Accessed 13 July 2020].
17 Rose SC, Bisson J, Churchill R, Wessely S. Psychological debriefing for preventing post-traumatic stress disorder (PTSD). Cochrane Database Syst Rev 2002;2:CD000560.

18 Tuckey MR, Scott JE. Group critical incident stress debriefing with emergency services personnel: a randomized controlled trial. Anxiety Stress Coping 2014;27:38-54.

19 Adler AB, Bliese PD, McGurk D et al. Battlemind debriefing and battlemind training as early interventions with soldiers returning from Iraq: Randomization by platoon. J Consult Clin Psychol 2009;77:928-40.

20 Hancock M. Plans for managing the coronavirus (COVID-19) outbreak in Leicester. UK Government, 2020. www.gov.uk/government/ speeches/local-action-to-tackle-coronavirus [Accessed 13 July 2020].

21 Carmassi C, Foghi C, Dell'Oste V et al. PTSD symptoms in healthcare workers facing the three coronavirus outbreaks: What can we expect after the COVID-19 pandemic. Psychiatry Res 2020;292:113312.

22 Williamson V, Murphy D, Greenberg N. COVID-19 and experiences of moral injury in front-line key workers. Occup Med (Lond) 2020;70:317-9.

23 O'Glasser A, Jaffe R, Brooks M. To tweet or not to tweet, that is the question. Semin Nephrol 2020;40:249-63.

24 World Health Organization. Managing the COVID-19 infodemic: Promoting healthy behaviours and mitigating the harm from misinformation and disinformation. WHO, 2020. www.who.int/news/ item/23-09-2020-managing-the-covid-19-infodemic-promotinghealthy-behaviours-and-mitigating-the-harm-from-misinformationand-disinformation.

25 Bendau A, Petzold MB, Pyrkosch L et al. Associations between COVID-19 related media consumption and symptoms of anxiety, depression and COVID-19 related fear in the general population of Germany. Eur Arch Psychiatry Clin Neurosci 2020, in press (doi: 10.1007/s00406-020-01171-6)

26 Gao J, Zheng P, Jia Y et al. Mental health problems and social media exposure during COVID-19 outbreak. PLOS ONE 2020;15:e0231924

27 Bernabeo EC, Holtman MC, Ginsburg S et al. Lost in transition: the experience and impact of frequent changes in the inpatient learning environment. Acad Med 2011;86:591-8.

28 Sturman N, Tan Z, Turner J. 'A steep learning curve': junior doctor perspectives on the transition from medical student to the healthcare workplace. BMC Med Educ 2017;17:92.

Address for correspondence: Dr Chandra Ohri, Institute for Lung Health, Glenfield Hospital, University Hospitals of Leicester NHS Trust, Groby Road, Leicester LE3 9QP, UK. Email: chandra.ohri@uhl-tr.nhs.uk 